\title{
EVALUATION OF THE PROPERTIES OF A NOVEL HYBRID ELASTOMERIC IMPRESSION MATERIAL
}

\author{
Heba Mohamed El-Amin Shalaby*
}

\begin{abstract}
The aim of this study was to evaluate and compare the flow of a polyether, and poly vinylsiloxane impression materials with a recently introduced vinyl polyethersilicone hybrid elastomeric impression material.

Materials and methods: The flow of the medium viscosities of the tested materials was measured using the shark fin Device. One minute after the start of mixing, the pin was released and the solid cylinder was allowed to sink into the impression under its own weight, where the material flowed up into the slit producing a shark fin-like layer of tested impression material. The heights of the shark fin were measured at 60 minutes after the start of mixing, using a digital micrometer with $0.001 \mathrm{~mm}$ resolution. One-way Analysis of Variance (ANOVA) was used for comparison between the three groups. Tukey's post-hoc test was used for pair-wise comparison between the groups when ANOVA test is significant.
\end{abstract}

Results: there was a statistically significant difference between fin heights of the three materials (P-value <0.001), where polyether showed the statistically significantly highest mean fin height (12.7), however the hybrid material showed statistically significantly lower mean fin height (7.6). Moreover addition silicone showed the statistically significantly lowest mean fin height (5.5).

Conclusion: Hybridization of addition silicon and polyether impression materials improved their flow.

KEY WORDS: Flow, vinylsiloxnether, shark fin test.

\section{INTRODUCTION}

Impression making and pouring are critical steps in the process of producing successful restorations in oral rehabilitation. Impression materials should reproduce hard and soft tissues around prepared and adjacent teeth in order to obtain biologically, me- chanically, functionally and esthetically acceptable restorations..$^{[1-3]}$

Elastomers or rubber base impression materials are the most widely used dental impression materials as secondary impressions all over the world in almost all the specialties of dentistry because it

\footnotetext{
* Professor Restorative Dentistry Department, Faculty of Dentistry, King Abdul Aziz University, Jeddah, KSA.
} 
meets most of the ideal requirements of impression materials ${ }^{[4]}$. Among the available elastomeric impression materials in the market, polyvinyl siloxanes and polyethers are used most frequently ${ }^{[5]}$.

As there is no impression material meets all of the ideal requirements, significant improvements have been made, vinyl-polyether hybrids were recently introduced which is the newest class of impression materials.

Impression material must have low viscosity toaccurately record impression details such as the interproximal spaces, deep restorative preparations, and gingival crevices. Low viscosity allows better flow of thematerial, which is important for accurately capturing fine details of the preparation. ${ }^{[6]}$

Normally, the flow properties of a material are characterized by rheological methods using e.g. a rheometer with plate-plate or plate-cone system.

Different measurements can be done to determine the yield point like the flow curve or a hardening curve. The calculation of the yield point is complex and difficult. A special knowledge about rheological parameters like storage modulus, loss modulus, tan delta and stress and strain parameters is necessary. For dental impression materials another simple test exists which is called sharkfin test ${ }^{[7]}$.

The shark fin test device was developed for testing the flow of impression materials. Several authors [8-9] published results with this shark fin testing apparatus. The initial design for this device simulated the flow of impression material from $1.5 \mathrm{~N}$ of applied force. The seating forces for elastomeric impress ionmaterials, as determined by Sotiriou and Hobkirk, reach as high as $10 \mathrm{~N} .^{[10]}$ To more closely simulate clinical forces; the device was modified by the addition of a $268.20 \mathrm{~g}(\sim 2.6 \mathrm{~N})$ weight on top of the metal caste. The weight increases the pressure applied to the material to more than $4 \mathrm{~N}$.
The weight more closely approximatesthe average pressure used.

\section{AIM OF THE STUDY}

The aim of this study was to evaluate and compare the flow of a polyether, and polyvinylsiloxane impression materials with a recently introduced vinylpolethersiliconehybrid elastomeric impression material in a trial to reach the one with theoptimum properties to be used as an accurate impression material.

\section{MATERIALS AND METHODS MATERIALS}

Three commercially available elastomeric impression materials were used in this study. The materials used are listed in Tables (1)

\section{Methods}

Materials were mixed using the corresponding mixing devices recommended by the manufacturers, together with the corresponding mixing tips. The flow of the medium viscosities of the tested materials were measured using the shark fin Device* (Figure 1). The shark fin device consists of upper and lower components: the lower component has a central depression surrounded by a split ring for incorporating about $7 \mathrm{ml}$ of the impression material.

The upper component consists of a hollow cylinder that fits exactly around the ring of the lower part and it has a central hole at its upper end. The upper component encloses a solid cylinder with a vertical slit at its lower end; this slit extends vertically up to a distance of $32 \mathrm{~mm}$. The slit was 2 wide at its outer end and extends horizontally in a V-shape to a distance of $1.8 \mathrm{~mm}$. The upper end of the solid cylinder has a narrow rod that extends through the central hole of the hollow cylinder.

The solid cylinder is suspended in its position by means of a horizontal releasing pin penetrating the 
TABLE (1) The three elastomeric impression materials used in the study.

\begin{tabular}{|l|c|c|c|}
\hline \multicolumn{1}{|c|}{ Impression materials } & Vinyl polyether silicone hybrid & Polyether & Addition Silicone \\
\hline Commercial name & Identium & ImpregumPenta & Express XT \\
\hline Batch \# & $100091-12$ & 70201136804 & 70201130872 \\
\hline Manufacturer & KETTENBACH Germany & 3M ESPE St. Paul, USA & 3M ESPE Germany \\
\hline ISO 4823-2000 Viscosity/Type & Medium/2 & Medium/2 & Medium/2 \\
\hline Working time (min:sec) & $2: 00$ & $2: 45$ & $1: 30$ \\
\hline Setting time (min:sec) & $5: 30$ & $3: 15$ & $2: 30$ \\
\hline Mixing device & Pentamix 2 & Pentamix2 & Garant dispenser \\
\hline
\end{tabular}

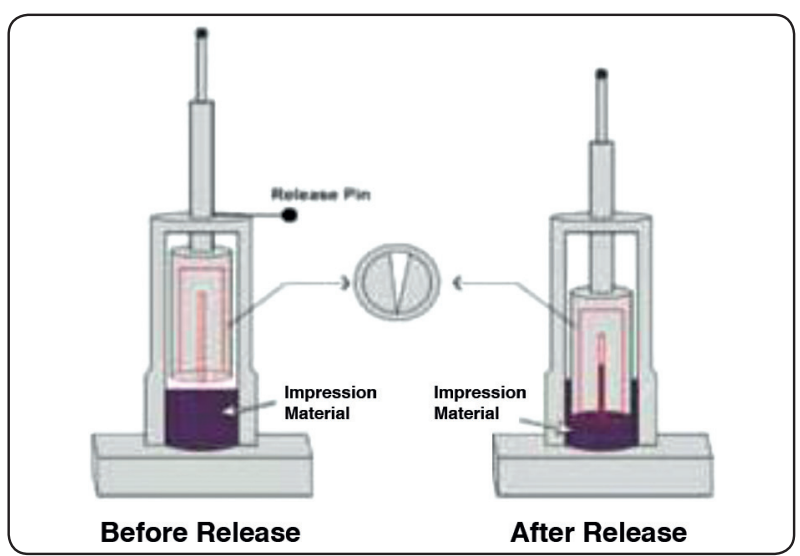

Fig. (1) Schematic drawing for shark fin device.

narrow rod just above the central hole. When the releasing pin is removed, the solid cylinder will be free to move downwards under its own weight (147 g) which simulates the pressure applied on the wash impression material during seating of the tray and sink into the material inside the split ring ${ }^{[11]}$.

\section{Shark fin sample preparation}

Each impression material was mixed and dispensed into the depression in the lower part, surrounded by the split ring, and leveled using a spatula. The upper compartment was then fitted on top, where the solid cylinder was suspended $10 \mathrm{~mm}$ above the level of the impression material by means of the pin.

One minute after the start of mixing, the pin was released and the solid cylinder was allowed to sink into the impression under its own weight, where the material flowed up into the slit producing a shark fin-like layer of tested impression material. After complete setting of the impression material the device was disassembled and the excess material was trimmed using a scalpel.

The heights of the shark fin were measured at 60 minutes after the start of mixing, using a digital micrometer with $0.001 \mathrm{~mm}$ resolution ${ }^{[7]}$.

For each sample, the mean of five measurements was taken and the height of the shark fin was calculated after subtraction of the height of the base). Five samples were made for each material.

Fin height=height of the whole specimen -height of the base

\section{Statistical Analysis}

Numerical data were presented as mean and standard deviation (SD) values. One-way Analysis of Variance (ANOVA) was used for comparison between the three groups. Tukey's post-hoc test was 
used for pair-wise comparison between the groups when ANOVA test is significant. Qualitative data were presented as frequencies and percentages. Chi-square (x2) test was used to compare between the groups. The significance level was set at $\mathrm{P} \leq$ 0.05 . Statistical analysis was performed with $\mathrm{IBM}^{*}$ SPSS** Statistics Version 20 for Windows.

\section{RESULTS}

Statistical analysis of the means and standard deviation of flow of the three tested impression materials are listed in (Table 2) and represented in (Figure 2).

From the table and the figure it can be noticed that there was a statistically significant difference between fin heights of the three materials (P-value $<0.001$ ), where Impregum showed the statistically significantly highest mean fin height (12.7), however Identium showed statistically significantly lower mean fin height (7.6). Moreover Express showed the statistically significantly lowest mean fin height (5.5).

TABLE (2) The mean, standard deviation (SD) values and results of One-way ANOVA test for comparison between fin height in $\mathrm{mm}$ for the three tested materials.

\begin{tabular}{|c|c|c|c|c|c|c|}
\hline \multicolumn{2}{|c|}{ Identium } & \multicolumn{2}{c|}{ Impregum } & \multicolumn{2}{c|}{ Express } & \multirow{2}{*}{$P$-value } \\
\cline { 1 - 5 } Mean & SD & Mean & SD & Mean & SD & \\
\hline $7.6 \mathrm{~b}$ & 1.2 & $12.7 \mathrm{a}$ & 0.6 & $5.5 \mathrm{c}$ & 0.2 & $<0.001^{*}$ \\
\hline
\end{tabular}

*: Significant at $P \leq 0.05$, Different letters are statistically significantly different

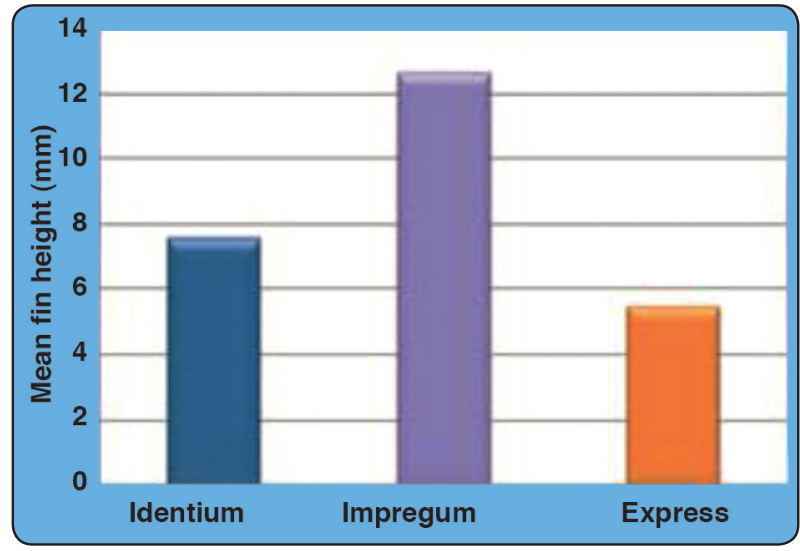

Fig. (1) Schematic drawing for shark fin device.

\section{DISCUSSION}

Results of the present study showed that the polyether impression material provided significantly greater flow followed by the hybrid impression and

a then the addition silicone impression material. This was consistent with the study carried by Lawson C. et al (201) ${ }^{[9]}$, Benchimol J. et al (2005) ${ }^{[12]}$, Broome JC. et al (2006) ${ }^{[13]}$, Klettke T. et al (2006) ${ }^{[8]}$ and German MJ. et al (2008) ${ }^{[7]}$

The ideal material would flow until it is ready to polymerize and then snap set. Unfortunately, most addition silicones have a slow setting time rather than a snap set. Therefore, viscosity increases soon after the material is mixed and continues to increase until the impression polymerizes.

Polyether impression materials provide increased flow at the end of the setting time with a sharper set time (i.e., polymerization occurs rapidly at the end of the setting time). This is especially important when making impressions of multiple abutments when all the working time is needed.

The new hybrid material used in this study has been reported by Baer Christopher J. (2011 $)^{[14]}$

\footnotetext{
* IBM Corporation, NY, USA.

**SPSS, Inc., an IBM Company.
} 
to possess good mechanical and flow properties, (double-snap) behavior where, the hybridization of the two types of impression materials allows for a long working time (a desired characteristic of polyethers) and a short set time (a desired characteristic of Polyvinylsiloxane). This (doublesnap) behavior occurs at the end of the working time. First, there is a noticeable viscosity snapfollowed by a cross-linkage snap that minimizes the opportunity for distortion upon removal and maximizes the accuracy of the impression ${ }^{[13]}$.

"Viscosity" describes the flow characteristics of an unset impression material. Materials with low viscosity have high flow and those with high viscosity have low flow. The viscosity of the material increases with the proportion of the fillers present, and it is affected by the shear force exerted on the material. The impression material can exhibit a decrease in viscosity in response to high shear stress and this is called shear thinning, thus, the viscosity varies in accordance with the shear stress. The higher the viscosity of the material, the more evident is the effect of shear thinning. This phenomenon is believed to be due to the small filler particle size ${ }^{[16]}$.

\section{CONCLUSIONS}

Hybridization of addition silicon and polyether impression materials improved their flow. The current study measured flow of impression materials in a dry condition. Clinically, impressions are taken usually in the presence of saliva. Therefore, future research could examine the ability of the impression material to flow on a wet surface. This study could be performed by modifying the shark fin testing method to allow testing flow in wet condition similar to the oral one.

\section{REFERENCES}

1. Perakis N., Belser U.C. and Magne P.: Final impressions: a review of material properties and description of a current technique. Int J Periodontics RestorativeDent; 24(2): 109117, 2004.
2. Thongthammachat S., Moore B.K., Barco M.T., Hovijitra S., Brown D.T. and Andres C.J.: Dimensional accuracy of dental casts: influence of tray material, impression material, and time. J Prosthodont; 11(2): 98-108, 2002.

3. Tjan A.H., Whang S.B., Tjan A.H. and Sarkissian R.: Clinically oriented evaluation of the accuracy of commonly used impression materials. J Prosthet Dent; 56(1): 4-8, 1986.

4. Anusavice KJ.: Phillips' Science of Dental Material .11th ed. St. Louis, Elsevier,2003.

5. Ferracane J.L.: Materials in Dentistry: Principles and Applications, 2nd ed, Lippincott, 2001.

6. Lowe R.A.: Mastering the art of impression making. Inside Dent;2(1): 38-39, 2006.

7. German M.J., Carrick T.E. and McCabe J.F.: Surface detail reproduction of elastomeric impression materials related to rheological properties. Dent Mater J; 24(7):951-956, 2008.

8. Klettke T., Ranftl D. and Kuppermann B.: Comparison of different types of impression materials. J Dent Res; 85B: 24-65, 2006.

9. Lawson N.C. , Cakir D., Ramp L.and Burgess J.O.: Flow profile of regular and fast setting elastomeric impression material using a shark fin testing device. J Esthet Rest Dent; 23(3): 171-176, 2011.

10. Ruff F., Jacobi A., Groten M. and Geis-Gerst: Hydrophilicity of elastomericnon aquous impression material during setting. Dent Mater J;21(2): 94-102, 2005.

11. Balkenhol M., Wöstmann B., Kanehira M. and Finger WJ.: Shark fin test and impression quality: a correlation analysis. J Dent; 35(5): 409-415, 2009.

12. Benchimol J., Perry R., Kugel G, and Halls M.: Flow of eightimpression materials with $2 \mathrm{~mm}$ slit after 25 seconds. JDent Res; 84A: 30-83, 2005.

13. Broome J.C., Burgess J.O. and Lawson N.C.: Flow of fast andregular set elastomeric impression materials. J Dent Re; 85C: 03-24, 2006.

14. Baer Christopher J.: Obtaining a high-quality impression with a new elastomeric material. Inside Dentistry; 7(6), 2011.

15. Vinyl polysiloxane impression materials: a status report. Council on Dental Materials, Instruments, and Equipment. J Am Dent Assoc; 120(5):595-6, 598, 600.1990.

16. Chai J., Pang I. : A study of the "thixotropic" property of elastomeric impression.Int J Prosthodont; 7(2): 155-8, 1994. 